\title{
Fat Grafting for Facial Contouring (Temporal Region and Midface)
}

Yun Xie, MD*, Ru-Lin Huang, MD, PhD, Wenjin Wang, MD, PhD, Chen Cheng, MD, Qingfeng Li, MD, PhD*

\section{KEYWORDS}

- Facial contouring • Fat graft • Midface • Temporal hollowing • Fat compartment

\section{KEY POINTS}

- Targeted restoration of the facial fat compartments allows for safe, reproducible, and effective outcomes of facial contouring.

- The authors advocate grafting fat tissue first into the deep plane (deep facial fat compartments) and then the superficial plane (superficial fat compartments), the medial part and then lateral part, and the upper side and then lower side.

- Better volume augmentation is based on more living fat graft transfer provided; thus, the authors use the 3L3M technique to get more living fat granules to fill the soft tissue.

Video content accompanies this article at http://www.plasticsurgery.theclinics.com.

\section{INTRODUCTION}

Facial volume loss in both bony and soft tissues results in hollowness and skin ptosis, which leads to concave facial contour, a deepened nasolabial fold, and midcheek groove, conveying an old and gaunt appearance. Autologous fat grafting is a well-accepted technique for soft tissue augmentation and has been advocated for facial contouring and body shape remodeling. There is no consensus in the literature, however, regarding the fat grafting technique, including the entry site for the cannula and the plane for fat placement. Historically, various fat grafting techniques have been developed and applied in clinical conditions and yield promising results, such as structure fat grafting (Coleman technique), ${ }^{1}$ the $3 \mathrm{M} 3 \mathrm{~L}$ technique, ${ }^{2}$ cell-assisted lipotransfer, ${ }^{3}$ and nanofat grafting. ${ }^{4}$ These techniques focus mainly, however, on the methodological aspects of fat harvesting, refining, and grafting skills in general.

Clinical observation and patient feedback reveal that long-term outcomes of fat grafting are diverse in different recipient sites, especially in the temporal region, one of the subunits with the lowest satisfaction rate after facial fat grafting. The authors believe the diversity of long-term outcomes of fat grafting is a result of different anatomic characteristics in different recipient sites that may directly influence grafted fat survival, neurovascular injury incidence, and fat tissue retention.

Based on these considerations and anatomic studies in the temporal region, ${ }^{5}$ midiface, ${ }^{6}$ and hand, ${ }^{7}$ the authors developed a concept of targeted fat grafting technique, which advocates targeted restoration of physiologic distribution and volume of fat compartments using anatomically

Disclosure Statement: The authors have nothing to disclose.

Shanghai Ninth People's Hospital, Shanghai Jiao Tong University School of Medicine, 19th Floor, Building 1, 639 Zhizaoju Road, Shanghai 200011, P.R. China

* Corresponding authors.

E-mail addresses: amiyayun@qq.com (Y.X.); dr.liqingfeng@yahoo.com (Q.L.) 
adequate cannula entry sites and injection planes. The authors' previous clinical studies have demonstrated effective and safety profiles in facial contouring, ${ }^{6}$ temporal hollowing augmentation, ${ }^{5,8}$ and hand rejuvenation ${ }^{7}$ and suggested more applications in other regions.

\section{PREOPERATIVE EVALUATION AND SPECIAL CONSIDERATIONS}

Each patient should be evaluated for general medical conditions in addition to some special preoperative evaluations, listed as follows:

1. Multiangles of standard photos-frontal, lateral, $45^{\circ}$ lateral, $90^{\circ}$ lateral, head up and head down, and some special angles-that can fully display the defect of face should be taken before operation. The photos can be printed to show patients where to fill and how thick they need to fill.

2. Three-dimensional (3-D) laser scan and 3-D volumetric analysis can be used to evaluate the symmetriy of the 2 sides of the face. The facial models are analyzed with corresponding analyzing software to quantify the volume discrepancy before graft, determining the quantity of the fat to inject.

3. For some pathologic soft tissue defects, such as radiation damage, scleroderma, and so forth, the skin may not have enough elasticity and be very tight or thin. The authors ask patient to stretch the skin for approximately 1 month to 3 months with small vacuum instruments or other tools to improve the recipient space.

4. CT scans may be used for Romberg disease patients at their first visit to make sure the atrophy degree of bone, muscle, fat tissue, and the occluding relation of their teeth.

The authors propose communicating well with each patient before operation for good compliance. Fat graft absorption is inevitable for every patient, especially during the first 3 months. To reach the final satisfactory results, patients may have injections 2 times to 3 times, with each time needing a 3-month to 6-month interval.

\section{SURGICAL PROCEDURE}

See Video 1. Before liposuction, the authors usually ask patients where the fattest part of their body is and for which part they most want to decrease the fat volume. Elderly women usually have fatty belly where abundant fat is easy to be suctioned. For young patients who do not have bigger belly, the authors prefer to get fat from their bilateral gluteal groove, which may have benefit for them to lift the most protruding point of gluteal and elongate the leg.

The second reason for harvesting fat from lower body position is because $\alpha-2$ receptors located in diet-resistant areas such as the lateral thighs buttocks, and abdomen. The following are the procedure of 3L3M fat graft technique.

1. Fat graft harvest: the total fat volume the authors use is no more than $100 \mathrm{~mL}$ for facial contouring purposes; thus, the authors prefer harvesting fat 'Manually' or 'syringe suction'. The composition of tumescent anesthetic fluid is $0.08 \%$ lidocaine plus $1: 500,000$ epinephrine. After injecting tumescent for several minutes, the authors use a $20-\mathrm{mL}$ syringe for hand liposuction. The authors keep $5 \mathrm{~mL}$ of air in the syringe before drawing back the Plunger pod and fixing it with a clamp or clip to create less negative pressure. The slower and gentler suction causes less oil from harvest fat. The authors may choose suction cannula with multiple side holes to do effective suction; the diameter of the side hole is chosen depending on the purpose of filling. For example, if volume augmentation and injecting to a deep layer are needed, a relatively larger hole, such as 2 $\mathrm{mm}$ to $3 \mathrm{~mm}$, may be chosen. If injecting superficial layer or performing some subtle injection, the authors prefer smaller side holes to do suction.

2. Fat graft process: after acquiring fat, the authors use saline to wash away lidocaine, epinephrine, cell debris, and red blood cells. Lidocaine and epinephrine, which contain the tumescent, are reported to reduce the viability of adipocytes. Then, the authors stand the syringe with fat and saline for a while, until the lowest part of liquid looks relatively clear, which means there are fewer small adipocytes suspension in the liquid part. Thin patients may have more fibers than fat patients. Fibers in the harvested fat are cut into pieces before low-speed centrifugation. The fat samples are centrifuged at low speed, less than $100 \mathrm{~g}$, for 2 minutes to $3 \mathrm{mi}-$ nutes. After centrifuge, the lowest liquid part is ejected out and then a 3-way or 2-way pipe connected to the $20-\mathrm{mL}$ syringe to transfer the middle layer of fat into a 2-mL or $1-\mathrm{mL}$ syringe. The top layer of oil is left in the syringe and used to lubricated inject cannula. The whole procedure is controlled at $25^{\circ} \mathrm{C}$ within 30 minutes to 60 minutes.

3. Recipient site preparation: Good design for patients should be done before surgery. 3-D 

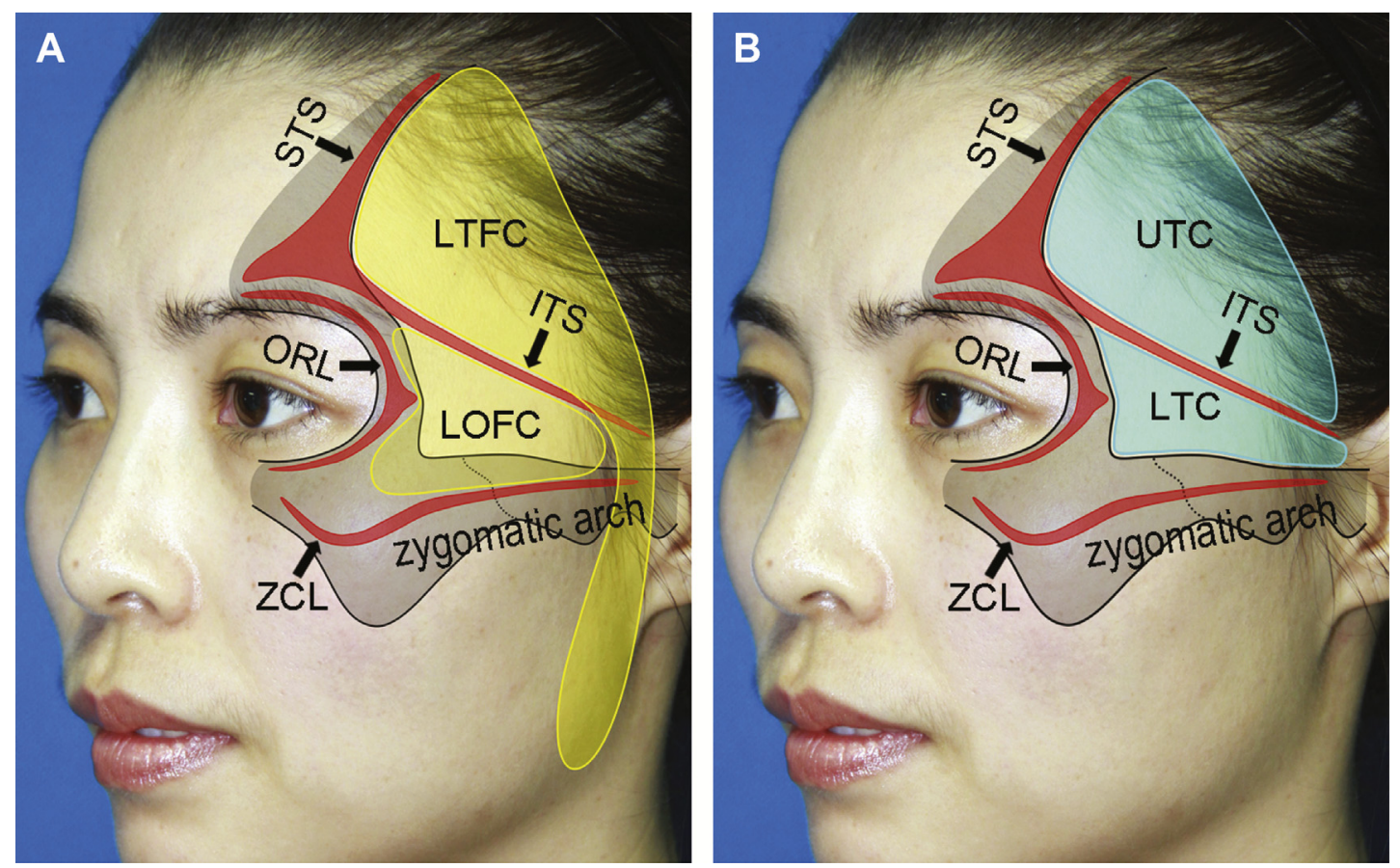

Fig. 1. Surface projection of fat compartments $(A)$ and septum compartments $(B)$ in the temporal region as demonstrated on a 37-year-old female patient. ITS, inferior temporal septum; LOFC, lateral orbital fat compartment; LTC, lower temporal compartment; LTFC, lateral temporal cheek fat compartmentORL, orbital retaining ligament; STS, superior temporal septum; UTC, upper temporal compartment; ZCL, zygomatic cutaneous ligament. (From Huang RL, Xie Y, Wang W, et al. Anatomical study of temporal fat compartments and its clinical application for temporal fat grafting. Aesthet Surg J. 2017;37(8):858; with permission.)

simulation or photos can help both surgeon and patients to make a decision. Make sure the recipient site is not injected with any other fillers, such as hyaluronic acid, collagen, and so on) or not use any enzyme to dissolve the fillers within 6 months. Make sure the skin has no infection (such as infectious acne) or ulceration, and the skin should have good elasticity to strut enough room for fat graft (Figs. 1-6).

Temporal hollowing augmentation can use the targeted fat grafting technique.Before fat grafting, the temporal hollowing region is marked by 4 borders:

1. Superiorly, the superior temporal line

2. Anteriorly, the lateral orbital rim

3. Inferiorly, the superior border of the zygomatic arch

4. Laterally, the temporal hair line

A small incision is made at the medial side of the head at the junction of the hairline and the temporal line. An 18G, single-holed, blunt-tipped infiltration cannula is inserted directly into this entry site and advanced along the surface of the skull until it enters the loose areolar tissue layer. Within this layer, the fat graft is placed into the upper temporal and the lower temporal compartments using a multiplane, multitunneling technique. The infiltration cannula is withdrawn from the space under the superficial temporal fascia and advanced into the subcutaneous fat layer in the temporal region. In this plane, the fat should be precisely grafted to the lateral temporal cheek fat and the lateral orbital fat compartments to correct the irregularities of the skin caused by the deep plane injection. To form a round and convex facial contour, the infiltration cannula also may be inserted into the subcutaneous fat layer in the forehead to adjust the contour between the temporal and forehead regions.

\section{Midface Fat Graft}

The recommend incision is presented as following. A paraoral commissure incision was made on the lip mucosa $1 \mathrm{~mm}$ to $2 \mathrm{~mm}$ adjacent to the oral commissure to avoid any unwanted scar formation. An injection cannula was placed beneath the oral mucosa, in the muscle, or subcutaneously in the first injection point to ensure accurate placement of fat in different layers in subsequent 


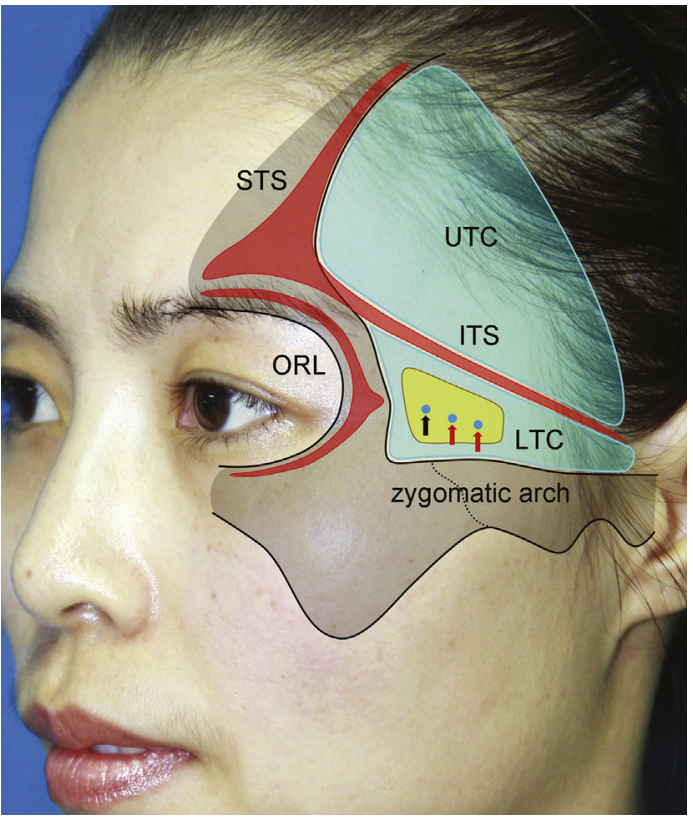

Fig. 2. Surface projection of the zone of caution as demonstrated on a 37-year-old female patient. The zone of caution is located in the lower temporal compartment at its anterior half, where the sentinel vein and branches of the middle temporal vessels perforate the temporal fascia and travel in the subcutaneous tissue layer (see arrows). ITS, inferior temporal septum; LTC, lower temporal compartment; ORL, orbital retaining ligament; UTC, upper temporal compartment; STS, superior temporal septum. (From Huang RL, Xie Y, Wang W, et al. Anatomical study of temporal fat compartments and its clinical application for temporal fat grafting. Aesthet Surg J. 2017;37(8):859; with permission.)

procedures. The second choice of incision is making a punch following the nasal labial fold with a $16 \mathrm{G}$ needle and then placing a cannula, with a diameter smaller than $1.2 \mathrm{~mm}$, that will not cause scarring.

The recommend injection technique is presented as followings. An 18G cannula connected to a 1-mL syringe was accurately placed beneath the lip mucosa or subcutaneously in the very beginning as required in subsequent procedures. When autologous fat was placed in the deep fat compartment, it was placed in the medial part of the deep medial cheek fat compartment by advancing the cannula between the mucosa and the orbicularis oris muscle. The root of the canine tooth was used as a bony marker to approach the medial part of the deep medial cheek fat compartment. The lateral part of the deep medial cheek fat compartment was approached by advancing the cannula in the same layer with the guidance of the first molar tooth and was

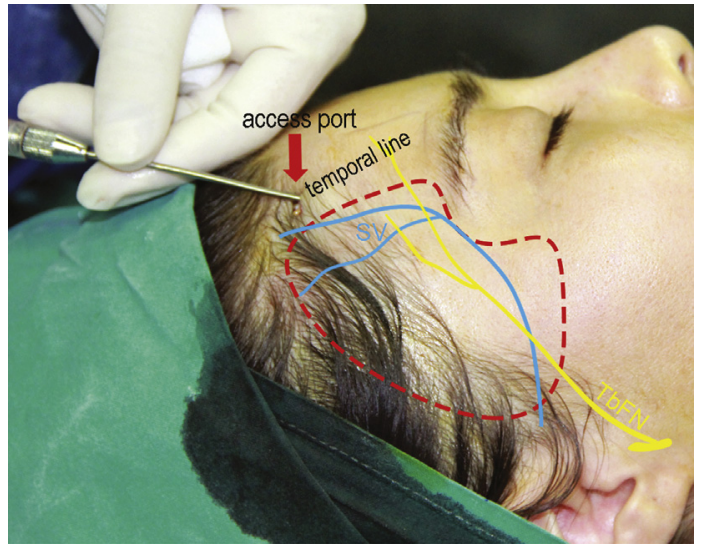

Fig. 3. The cannula entry site for temporal augmentation using fat grafting as demonstrated on a 37-yearold female patient. The entry site was placed medially to the junction of hairline and temporal line in an area that is relatively free of neurovascular structures. SV, sentinel vein; TbFN, temporal branch of the facial nerve. (From Huang RL, Xie Y, Wang W, et al. Anatomical study of temporal fat compartments and its clinical application for temporal fat grafting. Aesthet Surg J. 2017;37(8):860; with permission.)

advanced close to the maxilla. The medial part of the suborbicularis orbital fat compartment was also approached by advancing the cannula in this trajectory in a line, connecting the first molar and the lateral limbus of the ipsilateral cornea. Special attention was paid to avoid advancing the cannula too close to the maxilla when the cannula was around the level of the nasal ala. From this incision, the cannula was directed laterally to the superior part of the buccal fat pad from the superoanterior quadrant of the medial wall of the buccal fat pad by advancing the cannula close to the maxilla.

For augmentation of the middle and lateral cheek fat compartments, the fat was placed by directing the cannula to the lower one-third of the masseteric ligament where the fibrous structure is weak. Alternatively, another incision in the sideburn was used to avoid any unwanted injury to the blood vessels or nerves in the masseteric ligament (See Figs. 5 and 6).

\section{Case Demonstrations}

\section{Case 1}

A 46-year-old female patient with severe deep temporal hollowness of both temples underwent 2 autologous fat grafting procedures for targeted restoration of temporal fat compartments (Fig. 7). In the first procedure, $20.0 \mathrm{~mL}$ of autologous fat was injected in the right temple and $18.0 \mathrm{~mL}$ on the left side; 3 months after the first fat grafting procedure, the patient underwent the second fat 
A

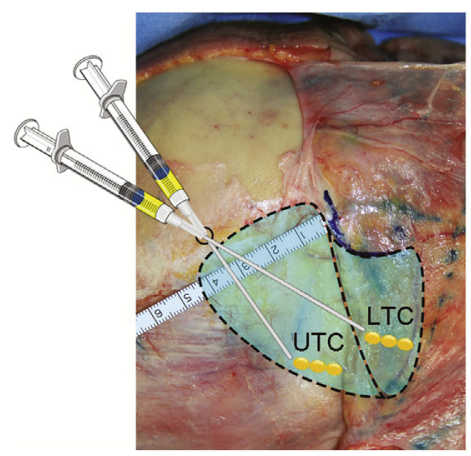

B

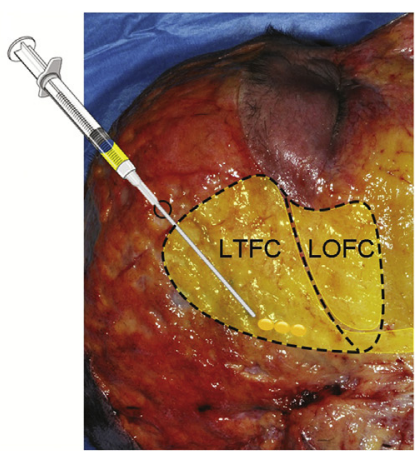

C

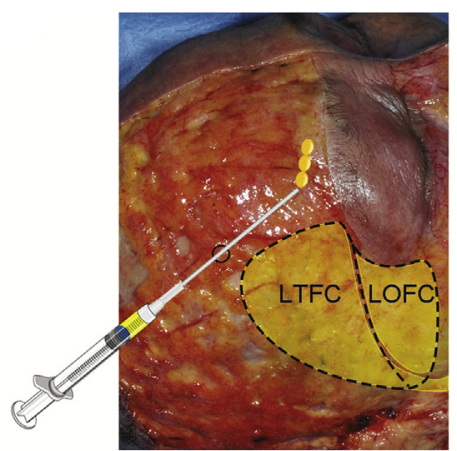

D

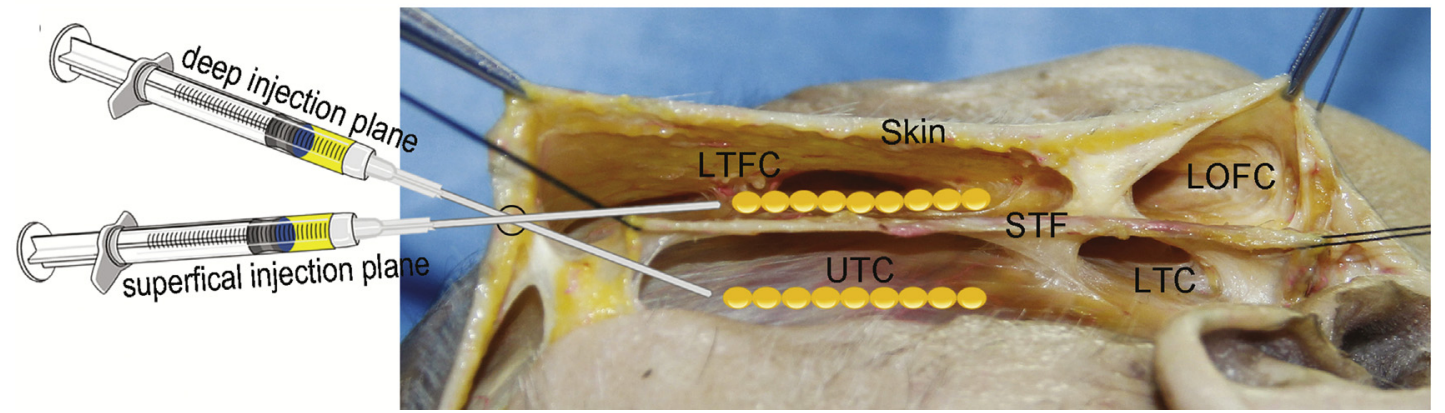

Fig. 4. Illustration demonstrating targeted fat grafting for temporal hollowing augmentation as demonstrated on a 72-year-old female cadaver. In this technique, the cannula entry is located medial to the junction of hairline and temporal line. The fat tissue is injected into the deep injection plane $(A)$ in the loose areolar tissue layer (UTC and LTC), to restore the majority of volumetric loss, followed by an injection into the superficial injection plane $(B)$ in the subcutaneous tissue layer (LFFC and LOFC), to achieve a smooth and round facial contour, and the transition zone $(C)$ in the subcutaneous tissue layer of the frontal region, to achieve a smooth and round facial contour. $(D)$ Illustration demonstrating the relationship of the superficial injection plane and the deep injection plane. LOFC, lateral orbital fat compartment; LTC, lower temporal compartment; LTFC, lateral temporal cheek fat compartment; STF, superficial temporal fascia; UTC, upper temporal compartment. (From Huang RL, Xie Y, Wang W, et al. Anatomical study of temporal fat compartments and its clinical application for temporal fat grafting. Aesthet Surg J. 2017;37(8):861; with permission.)

grafting procedure; $12.0 \mathrm{~mL}$ of fat was injected in each temple. At 12 months after the second fat grafting procedure, she presented only mild temporal hollowness in both temples.

\section{Case 2}

A 46-year-old female patient with moderate temporal hollowness of both temples underwent 1 autologous fat grafting procedure (Fig. 8); $18 \mathrm{~mL}$ of fat was grafted in each temple. Eighteen months after the fat grafting procedure, the patient appeared to have no hollowness in either temple.

\section{Case 3}

A 49-year-old female patient with temporal hollowness, obvious tear trough, and nasolabial folds of both sides underwent 1 autologous fat grafting procedure (Fig. 9). A total of $32 \mathrm{~mL}$ of fat was grafted in temporal region and midface. Twelve months after the fat grafting procedure, the patient appeared to have no hollowness in either temple. The volume of midface was augmented.

\section{POSTOPERATIVE CARE}

Immediately after the procedure, a compression dressing was applied to the area for 5 days to 7 days. For patients who were not satisfied with the augmentation outcome, a second surgical procedure was performed 3 months to 6 months after the initial procedure to improve the contour.

\section{EXPECTED OUTCOME AND MANAGEMENT OF COMPLICATIONS}

After the fat grafting procedure using the targeted fat compartment volume restoration technique, the curvilinear line on the temporal region and midface became smoother and less concave. A pleasing result of an elevated anterior projection 


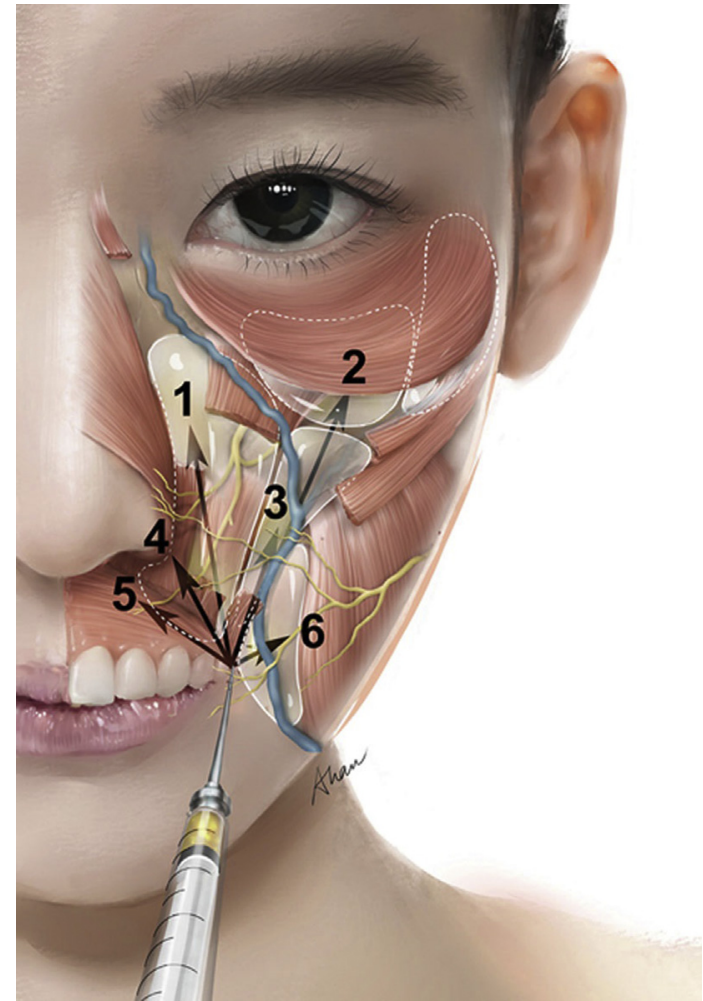

Fig. 5. Trajectory and sequence for deep fat compartment filtration. For fat placement in the deep cheek fat compartments, a specific sequence of fat placement is recommended: 1 , medial part of the deep medial cheek fat compartment; 2 , medial part of the suborbicularis oculi fat compartment; 3, lateral part of the deep medial cheek fat compartment; 4, lateral part of the nasal base; 5 , upper lip in the submucosa layer; and 6, superior part of the buccal fat pad. (Courtesy of Zhou Shuyang.)

of the temporal region and midface was obvious. Complication was rare in the authors' case series. Minor complications, such as chronic edema, headache, and skin irregularity, were observed but fully recovered without treatment. Overcorrection was the most frequent reported major complication and was treated by liposuction. No infection, calcification, fibrosis, and fat embolism were reported in the authors' case series.

\section{REVISION OR SUBSEQUENT PROCEDURES}

Considering the resorption of fat graft, the second surgery usually is done after 3 months to 6 months. Most patients may have only a 1-time injection. Only a few of them have fat graft 3 or more times.

\section{DISCUSSION}

Anatomic and clinical studies have shown targeted restoration of the volume of facial fat

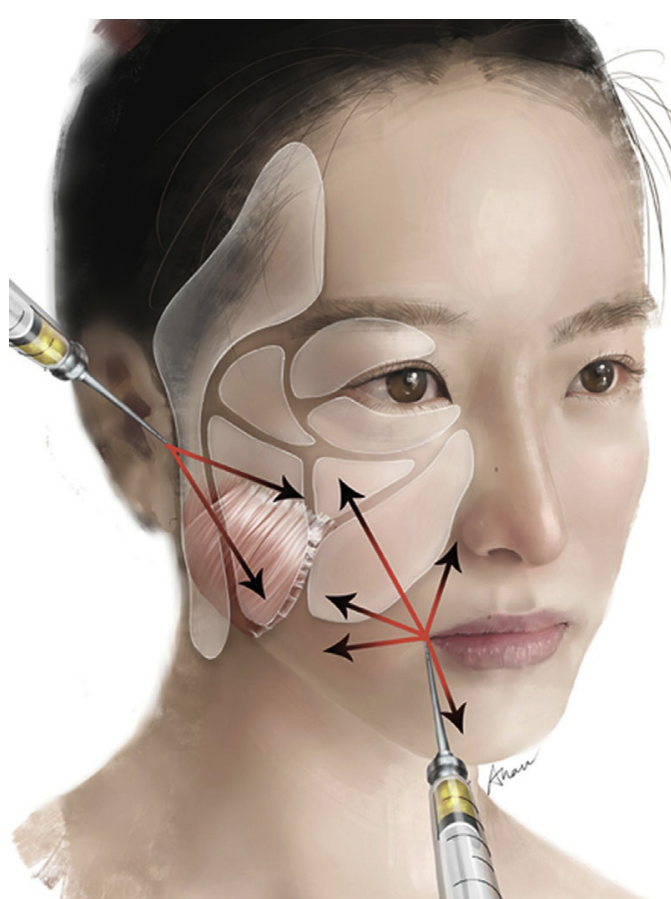

Fig. 6. Trajectory and sequence for superficial fat compartment infiltration through a paraoral commissure incision and an intraearlobe incision. The following sequence is recommended. First, direct the cannula laterally to approximately $1 \mathrm{~cm}$ lateral to the oral commissure, and turn upward toward the most prominent point of the zygoma and inject fat along the trajectory while withdrawing the cannula to create the upper half of the lateral border of the golden triangle (medial cheek fat compartment and nasolabial fat compartment). Second, direct the cannula laterally to approximately $1 \mathrm{~cm}$ lateral to the oral commissure and turn upward toward the mental tubercle and inject fat along the trajectory while withdrawing the cannula to create the lower half of the lateral border of the golden triangle. Third, direct the cannula to the nasolabial fat compartment and the medial cheek fat compartment medial and lateral to the lateral border of the golden triangle to create a smooth transition. Fourth, direct the cannula deep and along the nasolabial fold when necessary. For augmentation of the middle and lateral cheek fat compartments, another incision in the sideburn could be used as a complement to avoid any unwanted injury to the blood vessels or nerves in the masseteric ligament. (Courtesy of Zhou Shuyang.)

compartments by fat grafting not only restores the volume of facial fat compartments to a physiologic level and rebuilds a natural and youthful face but also avoids severe complications. Clinically, the 'temporal hollowing region' is different from a precise anatomic term, temporal fossa, and outlined by the superior temporal line, the 


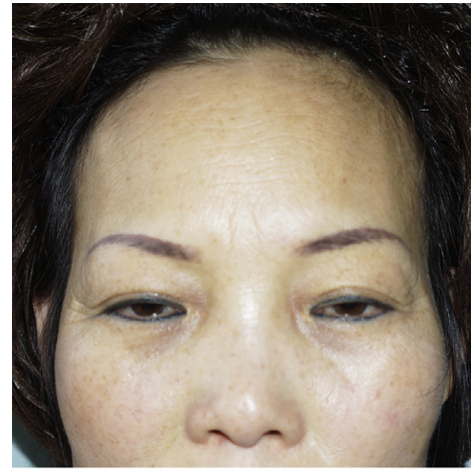

pre-op

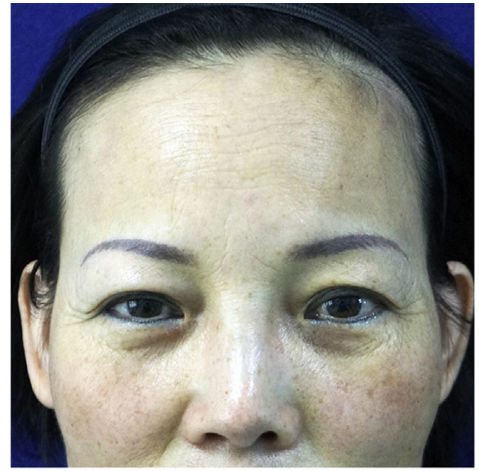

$3 \mathrm{~m}$ post-1st-op

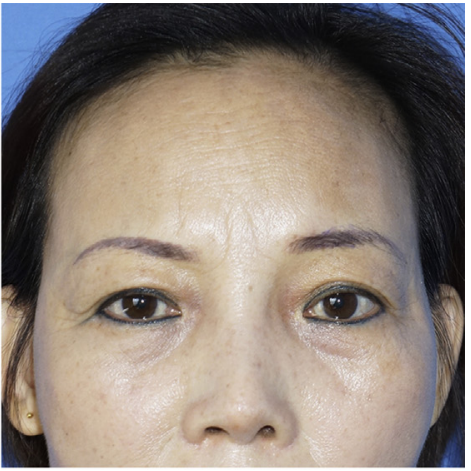

$12 \mathrm{~m}$ post-2nd-op

Fig. 7. Case 1: preoperative and postoperative photographs after fat grafting in the temple region. Right, preoperation; middle, 3 months post-1st-operation; right, 12 months post-2nd-operation.

temporal hairline, the zygomatic arch, and the lateral orbital rim. In this region, 4 separated fat compartments overlap in 2 planes: the lateral temporal cheek fat compartments and lateral orbital fat compartments, located in the superficial plane, and the upper temporal compartments and lower temporal compartments, located in the deep plane. Several important neurovascular structures pass through these fat compartments, including the perforators of the middle temporal vein, the sentinel vein, the frontal branch of the superficial temporal artery, and the temporal branch of the facial nerve. ${ }^{9}$ Therefore, the authors advocate that the fat tissue should be placed into the 4 temporal fat compartments through a unique entry site, which is located at the medial side of the intersection of the hairline and the temporal line.
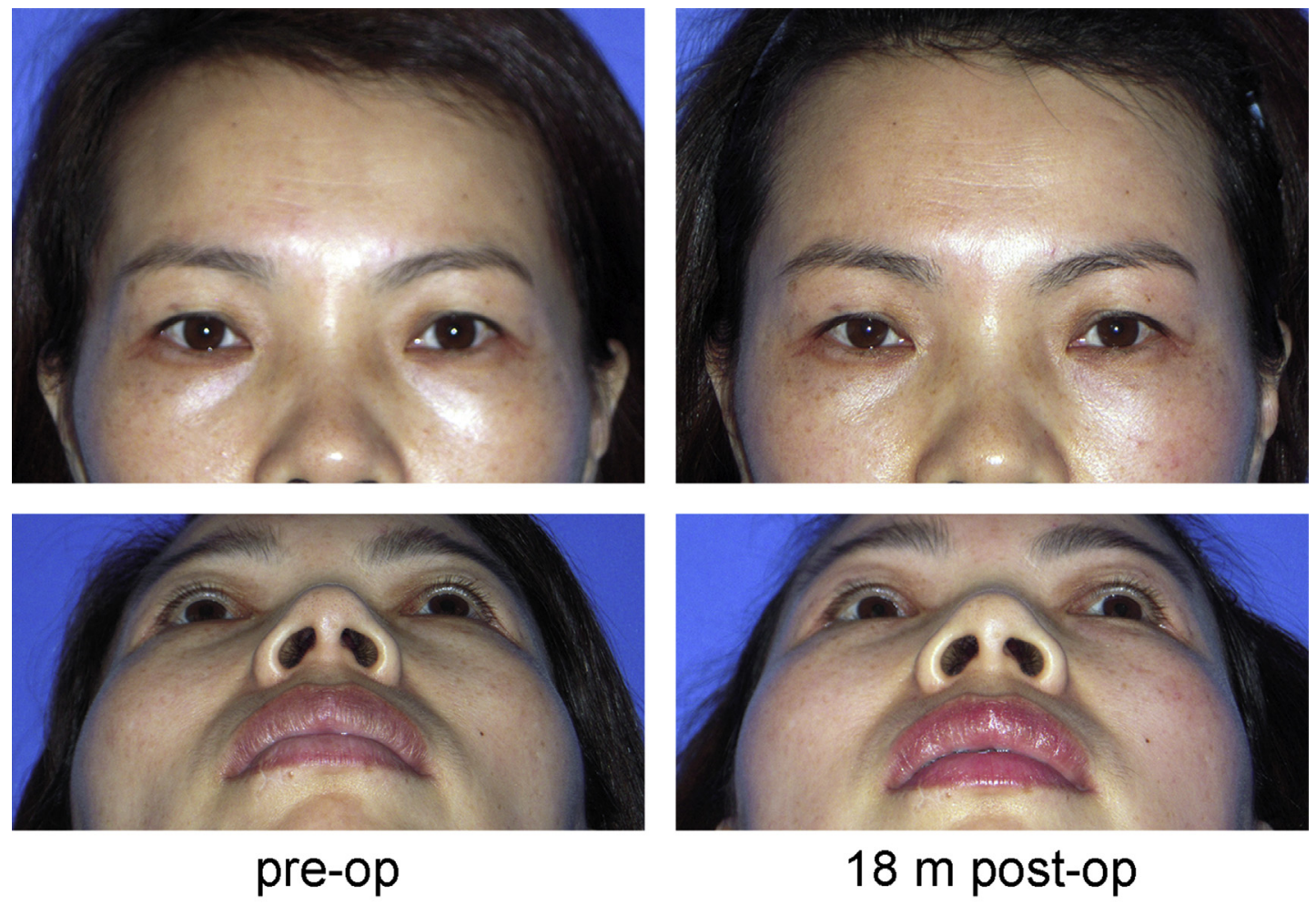

Fig. 8. Case 2: preoperative and postoperative photographs after fat grafting in the temporal region. Pre-op, preoperation; 18 m post-op, 18 months post-operations. 

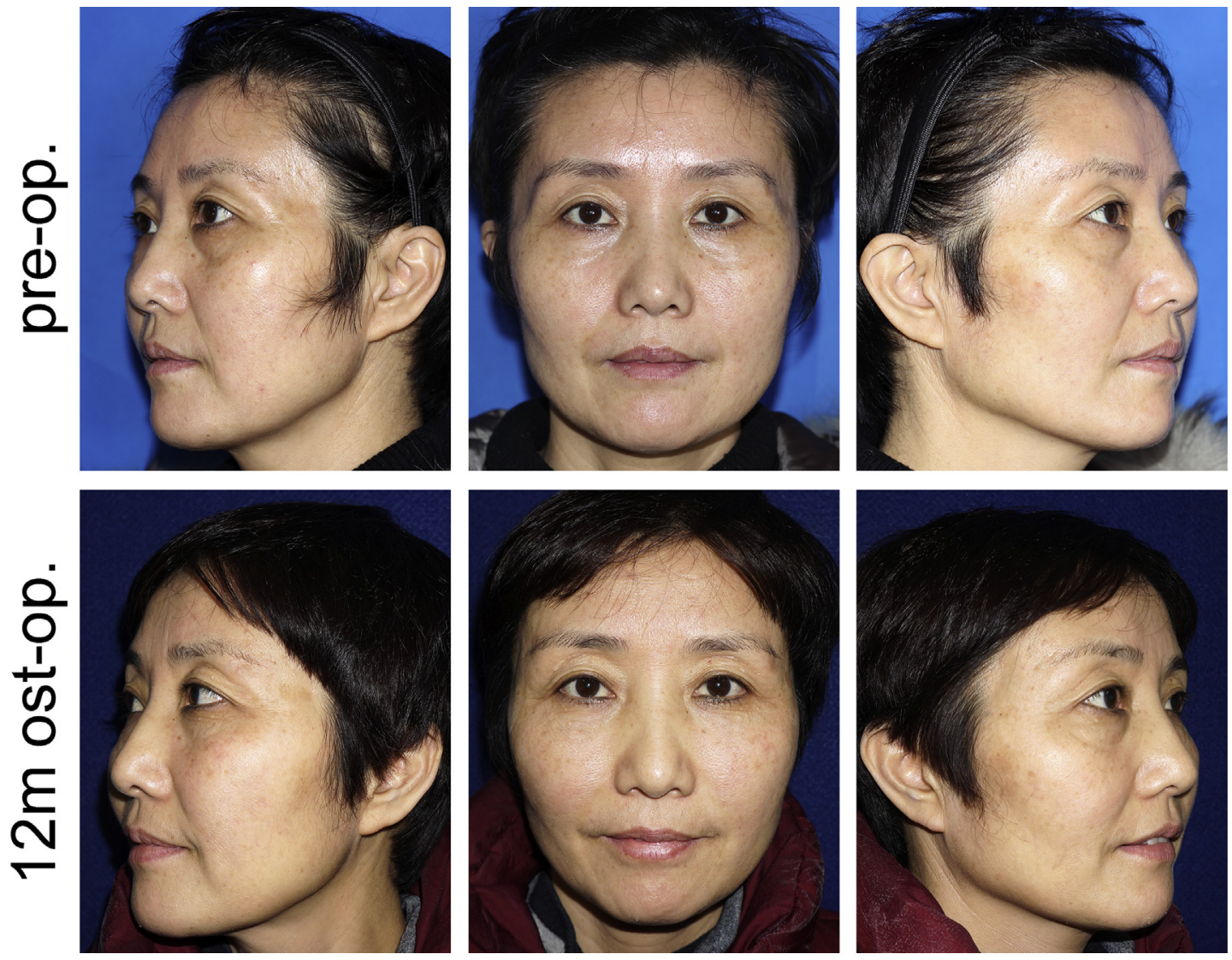

Fig. 9. Case 3: preoperative and postoperative photographs after fat grafting in the temporal region and midface.

In midface, the fat compartments are divided into superficial fat compartments (the superficial plane) and deep fat compartments (the deep plane). The deep fat compartments include the medial and lateral suborbicularis oculi fat compartments, the deep medial fat compartment, and the buccal fat compartment. These deep fat compartments support the fullness of the malar fat pad and play a major role in a youthful face. The superficial fat compartments include the nasolabial fat compartment, the cheek fat

\section{Box 1}

Recommended injection sequence of fat placement in the deep cheek fat compartments

1. Medial part of the deep medial cheek fat compartment

2. Medial part of the suborbicularis oculi fat compartment

3. Lateral part of the deep medial cheek fat compartment

4. Lateral part of the nasal base

5. Upper lip in the submucosa layer

6. Superior part of the buccal fat pad compartments, and the inferior orbital fat compartment. ${ }^{10}$ The authors' strategy for midface fat grafting is designed with the aim of fat placement in a multiplane and compartment-specific manner to achieve a state close to the physiologic fat distribution of a youthful face. Taking the advantages of hidden location and accessibility to different layers, the paraoral commissure incision is advocated for grafting fat tissue to the superficial fat compartments and the deep fat compartments in midface. Considering the changes of the deep and superficial fat compartments caused by aging and their physiologic function in

\section{Box 2}

Recommended injection sequence of fat placement in the superficial fat compartments

1. Superior half of the medial cheek fat compartment below the lid-cheek groove

2. Superior part of the nasolabial fat compartment below the midcheek groove

3. Superior part of the nasolabial fat compartment below the tear trough

4. Below the nasolabial fold when necessary 
constructing facial contour, a deep to superficial injection sequence is suggested. The recommended sequence of fat injection is shown in Boxes 1 and 2.

\section{SUMMARY}

Based on the authors' anatomic studies and clinical applications, the authors believe that compartment-based fat grafting is a safe and effective procedure for facial contouring for temporal region and midface.

\section{ACKNOWLEDGMENTS}

This work was supported by Project supported by the Funds for International Cooperation and Exchange of the National Natural Science Foundation of China (No: 81620108019); Natural Science Foundation of Shanghai (No: 17ZR1416500); Clinical Research Program of 9th People's Hospital (No: JYLJ004).

\section{SUPPLEMENTARY DATA}

Supplementary data related to this article can be found online at https://doi.org/10.1016/j.cps. 2019.08.008.

\section{REFERENCES}

1. Coleman SR. Facial augmentation with structural fat grafting. Clin Plast Surg 2006;33(4):567-77.
2. Xie Y, Li Q, Zheng D, et al. Correction of hemifacial atrophy with autologous fat transplantation. Ann Plast Surg 2007;59(6):645-53.

3. Yoshimura K, Sato K, Aoi N, et al. Cell-assisted lipotransfer for facial lipoatrophy: efficacy of clinical use of adipose-derived stem cells. Dermatol Surg 2008; 34(9):1178-85.

4. Tonnard P, Verpaele A, Peeters G, et al. Nanofat grafting: basic research and clinical applications. Plast Reconstr Surg 2013;132(4):1017-26.

5. Huang RL, Xie Y, Wang W, et al. Anatomical study of temporal fat compartments and its clinical application for temporal fat grafting. Aesthet Surg J 2017; 37(8):855-62.

6. Wang W, Xie Y, Huang RL, et al. Facial contouring by targeted restoration of facial fat compartment volume: the midface. Plast Reconstr Surg 2017; 139(3):563-72.

7. Zhou J, Xie Y, Wang WJ, et al. Hand rejuvenation by targeted volume restoration of the dorsal fat compartments. Aesthet Surg J 2017;38(1):92-100.

8. Huang RL, Xie Y, Wang W, et al. Long-term outcomes of temporal hollowing augmentation by targeted volume restoration of fat compartments in Chinese adults. JAMA Facial Plast Surg 2018;20(5):387-93.

9. Sadick NS, Dorizas AS, Krueger N, et al. The facial adipose system: its role in facial aging and approaches to volume restoration. Dermatol Surg 2015;41(Suppl 1):S333-9.

10. Rohrich RJ, Pessa JE. The fat compartments of the face: anatomy and clinical implications for cosmetic surgery. Plast Reconstr Surg 2007;119(7):2219-27 [discussion: 2228-31]. 